\title{
SEMIOLOGÍA RADIOLÓGICA EN PATOLOGÍA CEREBRAL DE EMERGENCIA
}

\author{
RADIOLOGICAL SEMIOLOGY IN EMERGENCY BRAIN PATHOLOGY \\ Alexander Román-Meza ${ }^{1,2, a, b, c,}$, Celso Huamán-Correa ${ }^{3,4, d, e, f}$
}

\section{RESUMEN}

La radiología es una gran herramienta en el diagnóstico de patologías encefálicas, especialmente en situaciones de urgencia y emergencia neurológicas, para descartar la presencia de una patología específica, y definir la localización y tamaño de la lesión, o esclarecer un diagnóstico inicial incierto. De esa forma, el conocimiento de sus principales signos, hallazgos y características es importante para realizar un examen adecuado, porque esto facilita la descripción de las imágenes obtenidas con una mayor rapidez y precisión permitiendo una labor óptima e inmediata del médico tratante en favor del paciente. Por ello, este artículo presenta una breve revisión de la semiología radiológica representativa obtenida en la literatura médica, donde las referencias fueron bibliografías e investigaciones, lo cual resulta conveniente para ubicar los signos que pudiese existir apenas fue obtenido el examen, para la futura terapéutica del paciente, así como la posibilidad de ampliación en el examen radiológico o sugerencia del tecnólogo médico de radiología en coordinación con el médico solicitante para garantizar la atención efectiva del paciente.

Palabras clave: Tecnología radiológica; Urgencias médicas; Encefalopatías; Lesiones traumáticas del encéfalo; Accidente cerebrovascular (fuente: DeCS BIREME).

\begin{abstract}
Radiology is a great tool in the diagnosis of encephalic pathologies, especially in situations of neurological urgency and emergency, to rule out the presence of a specific pathology, and to definite the location and size of the lesion, or clarify an initial uncertain diagnosis. In this way, the knowledge of its main signs, findings and characteristics is important to perform an adequate examination, because it facilitates the description of the images obtained with greater speed and precision allowing an optimal and immediate labor of the treating doctor in favor of the patient. Therefore, this article presents a brief review of the representative radiological semiology obtained in the medical literature, where the references were bibliographies and researches, which is convenient to localize the signs that could exist as soon as the examination was obtained, for the future therapeutic of the patient, such as the possibility of extension in the radiology exam or suggestion by the medical technologist of radiology in coordination with the requesting physician to guarantee the patient's care effective.
\end{abstract}

Key words: Radiologic technology; Emergencies; Brain diseases; Traumatic brain injuries; Stroke (source: MeSH NLM).

\footnotetext{
'Servicio de tomografía y resonancia, Hospital Nacional Edgardo Rebagliati Martins, EsSalud, Lima-Perú.

${ }^{2}$ Centro de excelencia de diagnóstico por imágenes de Clínica Internacional - Sede San Borja, Lima-Perú.

${ }^{3}$ Área de radiología de la escuela profesional de tecnología médica - Facultad de Medicina - Universidad Nacional Mayor de San Marcos, Lima-Perú. ${ }_{4}^{4}$ Policlínico ANCIJE - Red Desconcentrada Almenara - EsSalud, Lima-Perú.

a Licenciado en Tecnología Médica de Radiología; ${ }^{b}$ Especialista en resonancia magnética y tomografía computarizada; ${ }^{c}$ Magíster en Gerencia de Servicios de Salud, ${ }^{\mathrm{d}}$ Docente; ${ }^{\mathrm{e}}$ Licenciado en Tecnología Médica de Radiología; ${ }^{\mathrm{f}}$ Maestro en Educación con Mención en Docencia en el Nivel Superior. 


\section{INTRODUCCIÓN}

La radiología ha permitido constituir una gran herramienta de apoyo en el diagnóstico de patologías a nivel craneoencefálico en pacientes que ingresan por Emergencia en situaciones que requieren atención inmediata como la cefalea, el vértigo, el síncope, el coma, y las crisis epilépticas. Los exámenes radiológicos solicitados permiten confirmar o descartar la presencia de una patología específica, y ver la ubicación y extensión de una lesión, o esclarecer ante el hecho de no haber definido un diagnóstico inicial certero(1,2).

A partir de los cuadros de urgencia y emergencia neurológicas antes mencionados, existen diferentes enfermedades que son causales de estos cuadros. Específicamente nos referimos a ciertas patologías, que son vistas en Emergencia y son responsables de las situaciones antes mencionadas, y que de acuerdo a la clasificación de la patología cerebral comprende dos tipos: traumatismos craneoencefálicos y enfermedades cerebrovasculares ${ }^{(2,3,4)}$.

Definidas las patologías, se debe tener en cuenta sus signos radiológicos representativos, los cuales pueden encontrarse desperdigados en bibliografías, hemerografias y ciberespacio. En tal sentido, el conocimiento de los principales signos, hallazgos y características es importante porque permite considerar una realización adecuada del examen, así como facilitar la descripción de las imágenes obtenidas con una mayor rapidez y precisión aportando así al manejo óptimo e inmediato del médico tratante en favor del paciente ${ }^{(1,5,6)}$.

Cabe decir que las principales tecnologías radiológicas que se usan para esta valoración son:el radiodiagnóstico que pocas veces se utiliza actualmente, la tomografía computarizada en la mayoría de situaciones, y la resonancia magnética para casos específicos, los cuales se realizarán sin aplicación de sustancia de contraste endovenosa porque no se requiere su utilización en esta instancia, sin quedar descartado su uso en caso se opte por ampliar inmediatamente la prueba al momento tras el primer examen realizado ${ }^{(5,6,7)}$.

Se presenta un resumen de los signos radiológicos más representativos de la imagenología cerebral dada en emergencia, como una contribución para la ubicación del signo que pudiese existir apenas fue obtenido el examen, así como definir el manejo terapéutico a brindarse por parte del personal médico y enfermería, y la posibilidad de alguna ampliación inmediata o sugerencia del examen realizado por parte del personal tecnólogo médico de radiología en coordinación con el médico solicitante efectivizando la atención del paciente.

\section{REVISIÓN DE LOS PRINCIPALES SIGNOS RADIOLÓGICOS}

\section{Signo radiográfico de solución de continuidad ósea}

Este signo visto por radiografía simple de cráneo (RSC) constituye trazos definidos o líneas a nivel de la estructura ósea, que se denotan de un color negro (radiolúcido) en relación al tejido óseo circundante que se denota blanco (radiopaco). Las soluciones de continuidad ósea corresponden a fracturas, y de preferencia, tendrán una disposición de tipo lineal, aunque otros tipos morfológicos serán las fracturas por hundimiento, descalotamiento, o en mosaico (Figura 1). Hay que acotar que se pueden clasificar las fracturas según tipo anatómico en dos grupos: las de la bóveda y las de la base del cráneo, siendo las de difícil visualización en radiografía convencional las fracturas de la base ${ }^{(8,9,10,11)}$.

Cabe mencionar que la definición de este signo por una imagen radiográfica permite descartar su presencia en el paciente, debido a su alta especificidad y alto valor predictivo negativo (aprox. $94,9 \%$ y $83,1 \%$ respectivamente), es decir, tiene la alta probabilidad de encontrar un verdadero positivo, aunque se debe tomar ciertas reservas (condición y edad del paciente). Por ello, la radiografía debe ser utilizada sólo para corroborar la sospecha clínica que pudiese existir previamente en un paciente con exploración clínica normal. Siendo así, en otras situaciones la obtención de signos radiológicos será dará por tomografía computarizada $^{(8,9,10,11)}$.

\section{Signo tomográfico de fractura ósea}

Tal como fue en una imagen radiográfica, la tomografía computarizada permite ver trazos de fractura, pero con la ventaja de definir las que se presentan tanto a nivel de la bóveda como base de cráneo. Las fracturas se denotan como líneas de color negro o hipodensas, teniendo a su alrededor tejido óseo que se denotará blanco o hiperdenso. Todo esto será visible sólo en la ventana tomográfica adecuada (ventana ósea). Si es fractura lineal será trazo específico, si es deprimida los fragmentos se desplazarán internamente (Figura 1), y en ambos habrá aumento de partes blandas subyacente. Si es por descalotamiento se extiende por las suturas craneales, y si es en mosaico tendrá trazo tanto longitudinal como transversal, casos que pueden afectar arterias superficiales o senos venosos, o hasta duramadre, con posibilidad de presentarse neumoencéfalo como una secuela ${ }^{(8,12,13,14)}$. 


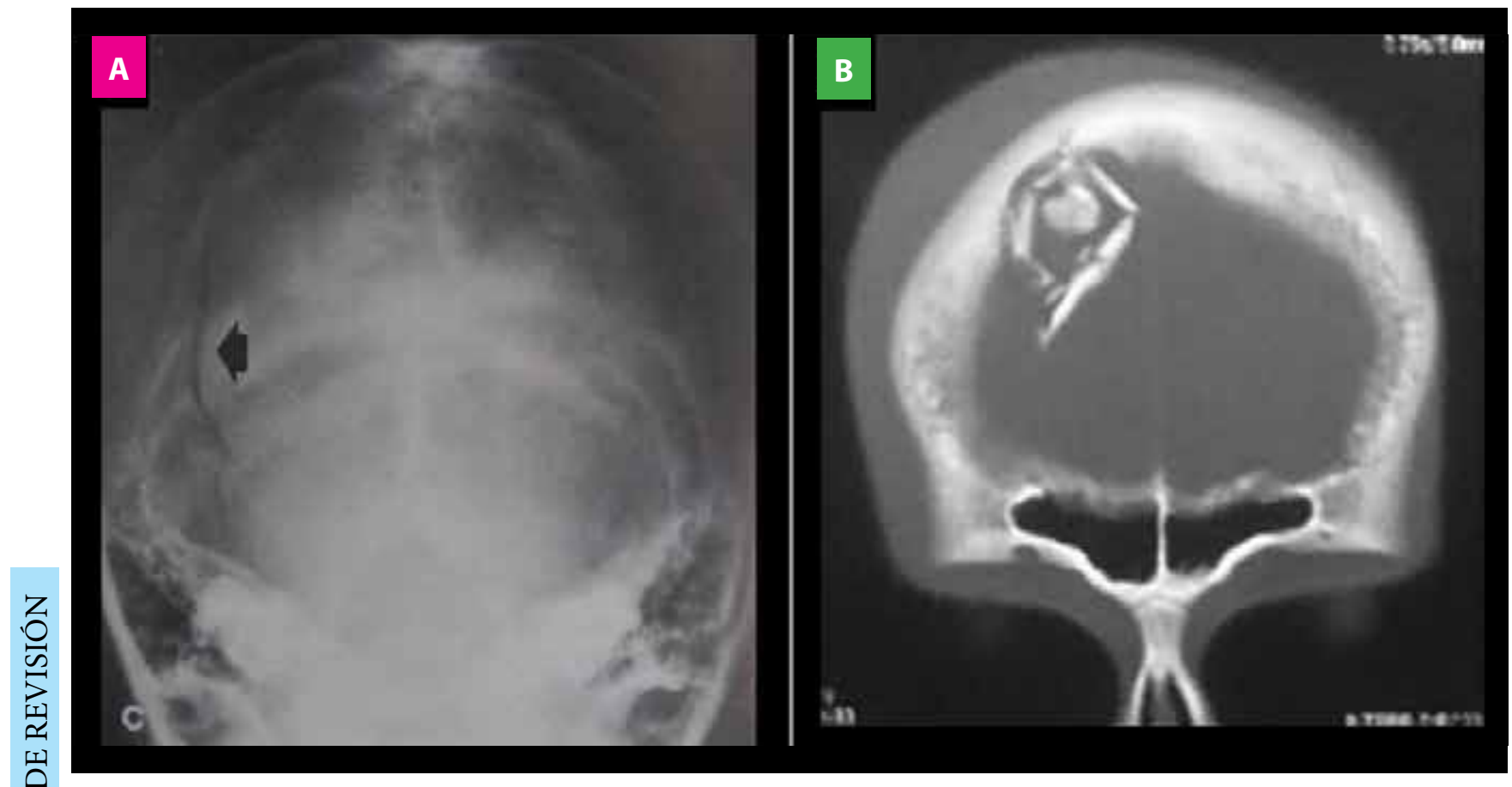

Figura 1. Signo radiográfico de solución de continuidad ósea (A) y signo tomográfico de fractura ósea (B).

\section{Signo de masa extraaxial biconvexa}

Es la representación clásica de un hematoma epidural, el cual se ubica en relación a la zona craneal donde se haya presentado trauma, a nivel del cerebro subyacente, con espacio subaracnoideo comprimidos, denotando una doble convexidad (hacia el parénquima cerebral como también hacia la calota craneal), desplazando la interfase entre sustancias gris y blanca.

Se presenta en un $85-90 \%$ de casos traumáticos, produciéndose en el punto de impacto, y su visualización se realiza preferentemente por tomografía computarizada (Figura 2), donde un $2 / 3$ de los casos se muestra hiperdenso y $1 / 3$ mixto (con contenido hiper/hipo), mientras que en resonancia magnética por lo general se mostrará isointenso con la cortical en la mayoría de secuencias con una línea negra entre hematoma y parénquima (duramadre desplazada ${ }^{(13,15,16,17)}$.

\section{Signo de colección extraaxial semilunar}

Es la representación clásica de un hematoma subdural, que denota una colección semilunar sobre el hemisferio cerebral, con puntos de líquido cefalorraquídeo en los surcos comprimidos y desplazados internamente respecto a la bóveda craneal, que puede cruzar suturas, pero no inserciones durales. Visualizado básicamente por tomografía computarizada, un $60 \%$ es hiperdenso (Figura 2), y un $40 \%$ es mixto (con contenido hiper/ hipo) que denota hemorragia activa o aracnoides desgarrada, todo esto sobre todo si el hematoma tiene días o a lo mucho pocas semanas de presentación. Conforme el hematoma tenga mayor tiempo de evolución, de semanas a meses, puede presentar septos hiperdensos (morfología trabecular) o verse hiperdenso e hipodenso en las zonas más baja y más alta del hematoma respectivamente, es decir, doble contenido (morfología separada) ${ }^{(13,16,18,19)}$.

\section{Signo de hemorragia cerebral parcheada}

Es el signo característico de las contusiones cerebrales, producida a partir de un traumatismo cráneo encefálico previo, donde las circunvoluciones impactan con el hueso, siendo de localización más frecuente a nivel anteroinferior en lóbulos temporales, en corteza perisilviana, y a nivel anteroinferior en lóbulos frontales (Figura 2). Se asocia en un 35\% de casos a fracturas craneales y en un $70 \%$ de casos a hematomas subgaleales, donde su visualización inicial es por medio de tomografía computarizada, donde se muestra una cortical hipodensa con una o múltiples lesiones hiperdensas que representan las contusiones, mientras que una resonancia magnética definirá con mayor detalle la extensión de la lesión donde la cortical se mostrará edematosa ${ }^{(19,20,21,22)}$.

\section{Signo de hematoma subgaleal}

Es el clásico hematoma de tejidos blandos dada a partir de un simple golpe en la cabeza, donde se produce extravasación celular por debajo de la aponeurosis de la 
región craneana, que tiende a permanecer fluctuante y líquida, y que se ubica en los límites de sus inserciones, por delante hasta la región supraorbitaria, a los lados por las regiones temporales y por detrás hasta el nivel de la nuca. Puede formarse sin haber lesión ósea, o con fracturas no desplazadas. El hematoma que invade el tejido conjuntivo laxo separa la galea aponeurótica del periostio y puede atravesar las suturas. Este signo se hace notorio gradualmente entre $12-72$ horas después de ocurrido el trauma, pero puede notarse más tiempo en casos graves. Su visualización se da mediante una tomografía cerebral, con ventana parenquimal, donde se observará como una hiperdensidad adherida externamente a la calota (Figura 2) $)^{(5,7,23,24)}$.

\section{Signo de hemorragia intraparenquimal}

Es la representación del contenido hemático a nivel del parénquima cerebral en general que pueden ser lobares o profundos, y estos últimos a su vez serán principalmente a nivel de los ganglios basales (si está asociada a crisis hipertensivas), tálamo, tronco encefálico, cerebelo. Este hematoma tendrá una forma ovalada, con límites por lo general precisos, con hiperdensidad en su interior y edema perilesional, los cuales de ser irregulares indicarán mal pronóstico, y en todos los casos con el respectivo efecto de masa, todo esto visualizado rápidamente mediante tomografía computarizada, más aún si la hemorragia tiene alto contenido sanguíneo o es de gran extensión (Figura $3^{(20,25,26,27)}$.
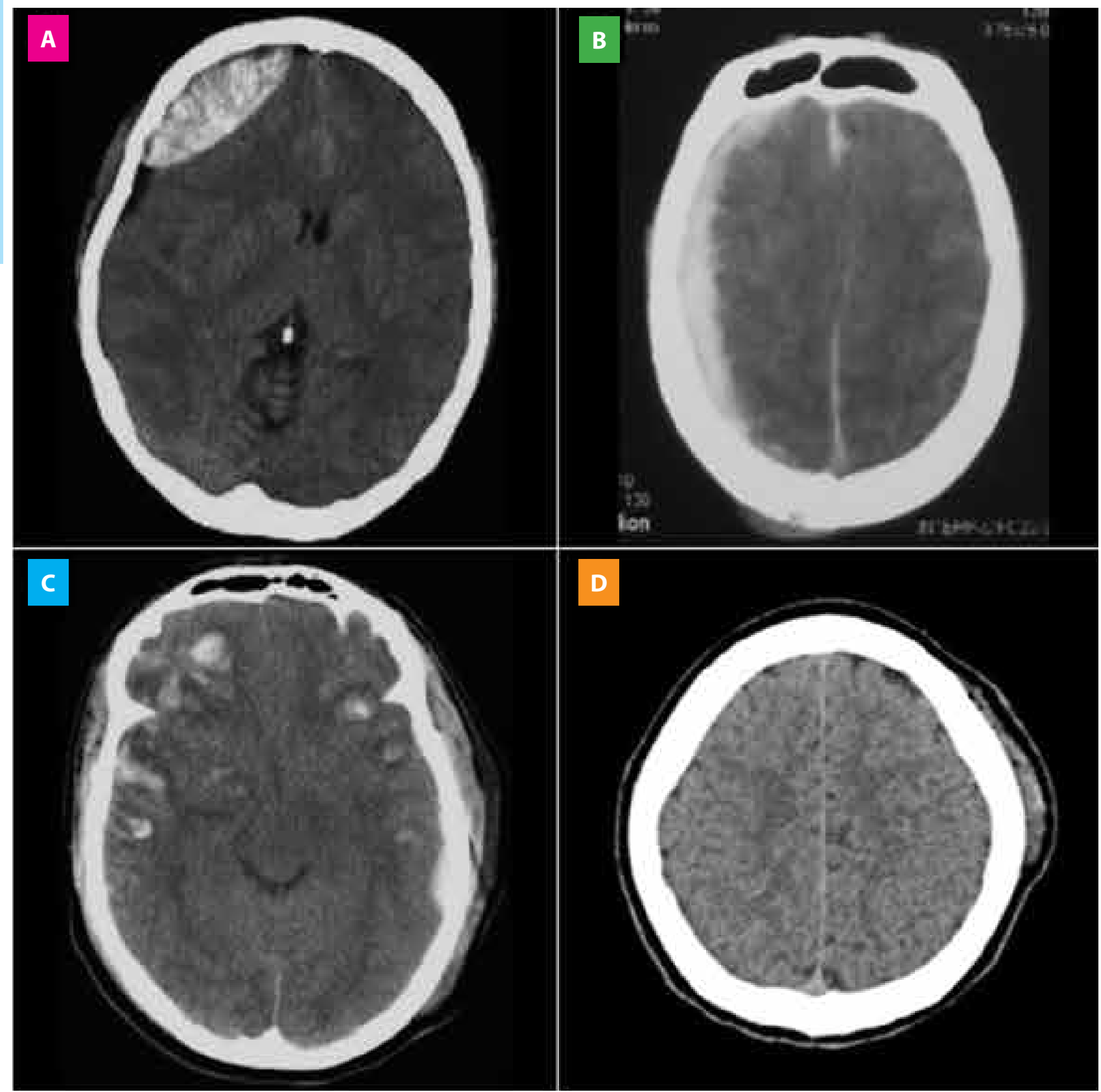

Figura 2. Signo de masa extraaxial biconvexa (A), signo de colección extraaxial semilunar (B), signo de hemorragia cerebral parcheada (C) y signo de hematoma subgaleal (D). 


\section{Signo de hemorragia subaracnoidea}

Es la representación del contenido hemático a nivel de los espacios subaracnoideos y sus expansiones dadas alrededor de tronco encefálico, incisura tentorial y agujero magno, denominadas cisternas cerebrales. Este hallazgo se observa justamente como hiperdensidad de cisternas basales y surcos de la convexidad y extendiéndose por los ángulos pontocerebelosos, plexos coroideos y sistema ventricular (Figura 3). La extravasación aguda de sangre puede deberse a fugas

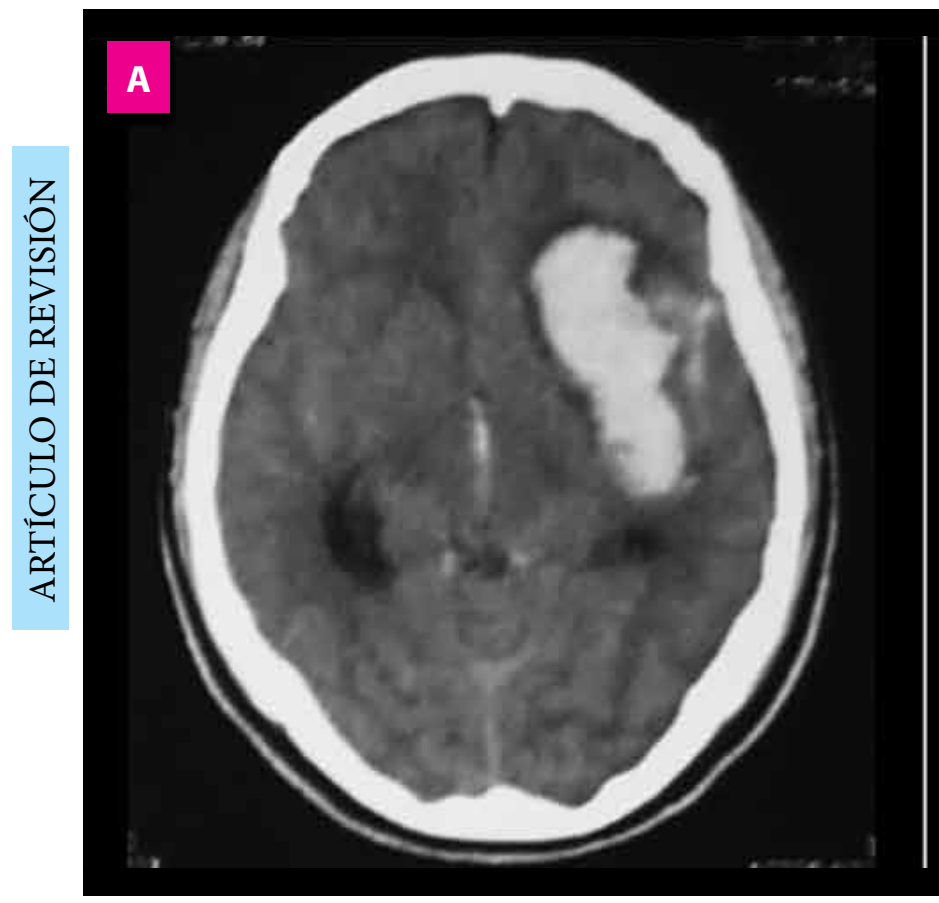

arteriales o a desgarros venosos. Las causas potenciales son los traumatismos, los aneurismas reventados, las malformaciones vasculares y la angiopatía amiloide. La hemorragia subaracnoidea si es traumática es adyacente a las contusiones y se observa mayor cantidad de contenido hemático en los surcos de convexidad que en las cisternas basales, mientras que, si la hemorragia es aneurismática, habrá mayor contenido en cisternas, requiriéndose luego alguna prueba angiográfica para precisar la lesión ${ }^{(20,24,25,28)}$.

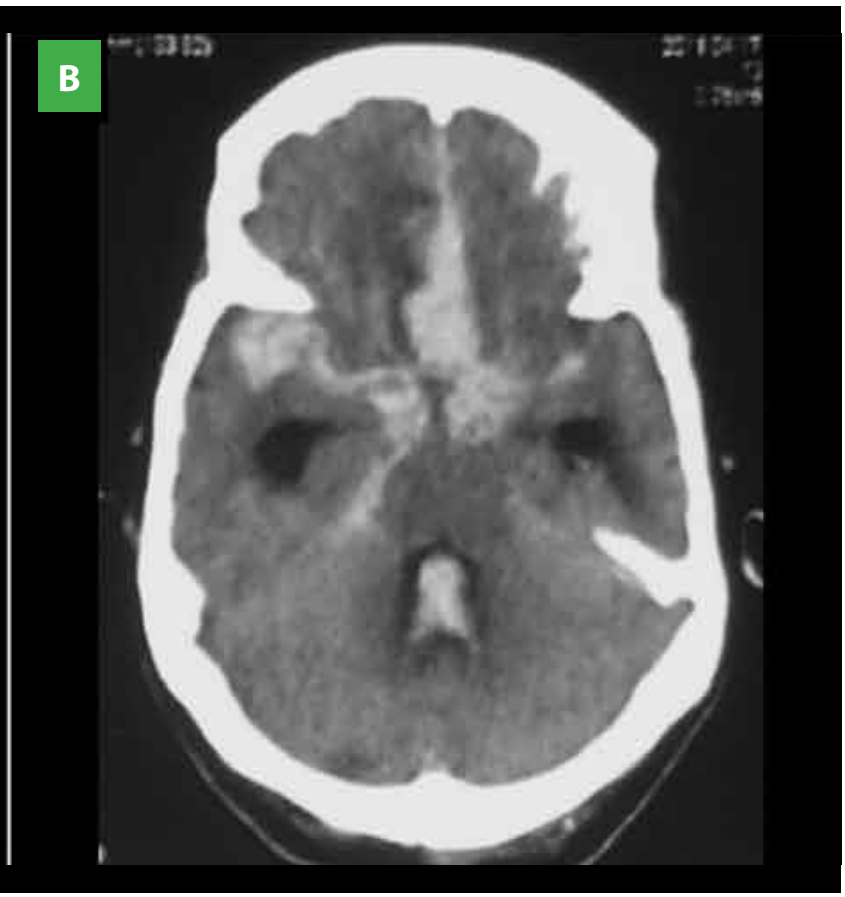

Figura 3. Signo de hemorragia intraparenquimal (A) y signo de hemorragia subaracnoidea (B).

\section{Signo de hipodensidad del núcleo lenticular}

Es la representación de isquemia aguda del territorio lenticuloestriado, denotándose por tomografía computarizada como un obscurecimiento en su interior por edema citotóxico, dado sobre todo por infartos en territorio cuya irrigación está dada por la arteria cerebral media. Al producirse esta hipodensidad se afecta tanto el globus pálido como el putamen, aunque el primero se afecta de forma más temprana, y esto puede observarse en tan solo dos horas de comenzado el evento isquémico (Figura 4). Hay que acotar que esta representación se puede observar de forma específica a las seis horas junto con pérdida de diferenciación de sustancia blanca y gris en la corteza cerebral| ${ }^{(5,29,30,31)}$.

\section{Signo de arteria cerebral media hiperdensa}

Es un signo precoz de enfermedad cerebrovascular sea de isquemia o hemorragia, visible en la tomografía sin contraste, descrita inicialmente en 1981 por D.H. Yock, que corresponde a la alta densidad a nivel de la arteria cerebral media en comparación con la arteria contralateral, siendo su localización más frecuente el primer segmento, dada por la oclusión de un émbolo o trombo, donde su densidad radiológica será mayor que la sangre circulante (80 y 40 Unidades Houndsfield respectivamente) por la mayor cantidad de proteínas y fibrina, con un suero en menor proporción (Figura 4). Aunque este signo tiene alta especificidad, casi del $100 \%$, su sensibilidad es relativamente baja, aprox. $30 \%$, siendo ocasionalmente un signo falso positivo, especialmente cuando si se presenta de forma bilateral y en pacientes que tengan adicionalmente calcificación de arterias o hematocritos altos, así como pacientes sin sintomatología con deshidratación o poliglobulia. Además, la apariencia de este vaso puede ser hiperdensa en pacientes que presentan calcificaciones vasculares o con enfermedad arteriosclerótica(5,25,29,30).

\section{Signo de la cinta cortical o insular}

Es el signo de hipodensidad de la corteza a nivel del ribete del lóbulo de la ínsula, que conlleva a una pérdida de distinción entre corteza insular y cápsula externa/ 
extrema, con pérdida de diferenciación entre sustancia blanca y gris (Figura 4). Este signo se presentará porque un edema citotóxico dado a nivel de la corteza de insular es susceptible a los cambios tempranos e irreversibles del evento cerebrovascular isquémico, especialmente por ser parte del territorio irrigado por la arteria cerebral media, cuya capacidad es menor para suplir la circulación mediante vasos colaterales desde las arterias cerebral anterior y cerebral posterior ${ }^{(5,29,30,31)}$.

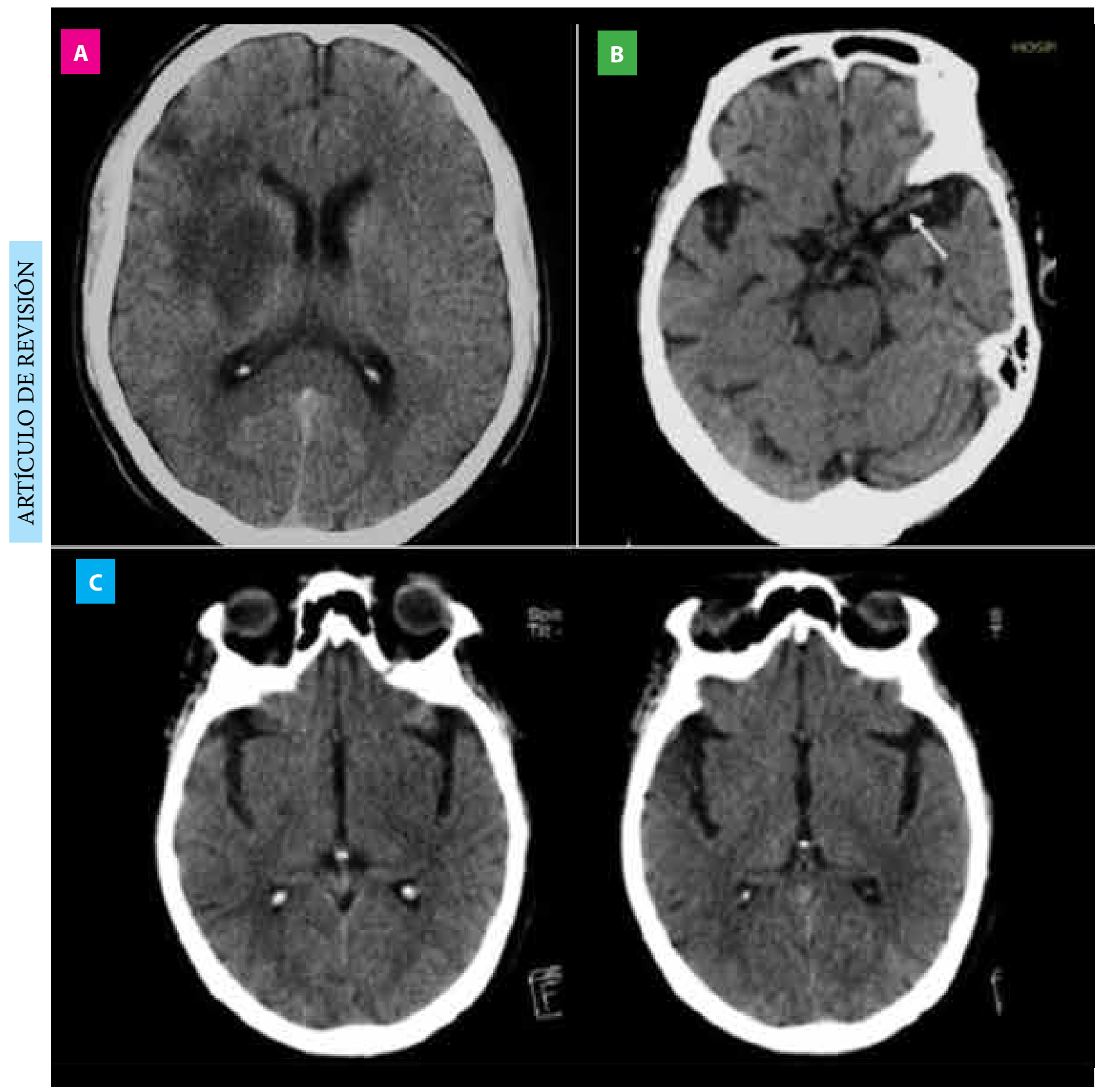

Figura 4. Signo de hipodensidad del núcleo lenticular (A), signo de arteria cerebral media hiperdensa (B) y signo de la cinta cortical e insular (C).

\section{Signo de restricción de difusión o de isquemia precoz}

Es la representación clásica del edema citotóxico intracelular dada por resonancia magnética situación donde la movilidad del agua está restringida y que se da principalmente en la isquemia cerebral, y cuya detección precoz en este examen se realiza en poco tiempo mediante la secuencia de difusión. Esta secuencia, que es ecoplanar, detecta el movimiento aleatorio o browniano de las moléculas del agua en diferentes direcciones de gradiente del campo magnético, que de estar restringido como en la isquemia, será detectable rápidamente ${ }^{(2,4,32,33)}$.

Esto será de ayuda cuando la tomografía computarizada no brinde ningún signo a pesar de los signos clínicos 
existentes, donde esta secuencia detectará cambios muy tempranos entre los 30 minutos y las 6 primeras horas, valorando tanto la imagen de difusión como su mapa ADC que muestra de forma pura el contraste basado únicamente en las diferencias de difusión sin tener en cuenta el contraste T2, es decir, a nivel de la zona donde se produzca edema citotóxico, se denotará con un color blanco brillante (hiperintenso), y su mapa de $A D C$ va a detectar lo mismo, pero se va a denotar de color negro (hipointenso), situación que no es diferenciable en una secuencia T1 (Figura 5) $)^{(2,4,32,33)}$.

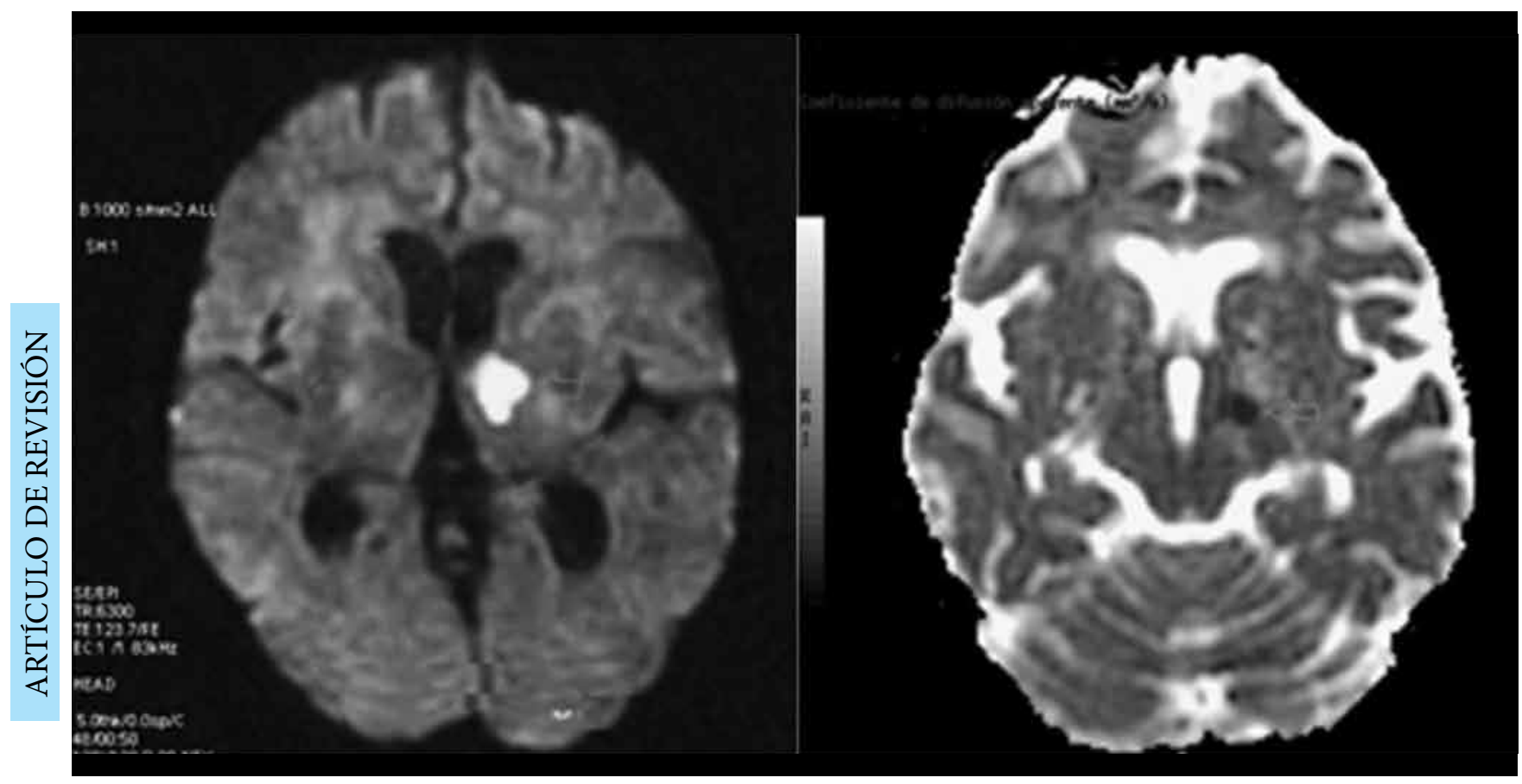

Figura 5. Signo de restricción de difusión o de isquemia precoz.

\section{CONCLUSIÓN}

Revisando información obtenida en algunas investigaciones como bibliografía referencial, se concluye que mediante la imagenología diagnóstica se puede definir signos, hallazgos y características radiológicas que denotan claramente la existencia de alguna patología a nivel craneoencefálico que haya sido causante de algún cuadro de urgencia o emergencia neurológica, que serán por ende patognomónicos. De esta forma, el conocimiento de la semiología radiológica permitirá describir rápidamente si estas existiesen tanto para el manejo terapéutico como para la ampliación inmediata o sugerencia por parte del personal tecnólogo médico de radiología en coordinación con el médico solicitante efectivizando la atención brindada al paciente que ha ingresado por emergencia.
Contribuciones de autoría: ARM concepción y diseño del artículo, recolección de información, análisis de referencias, y redacción del manuscrito; $\mathrm{CHC}$ planeación y asesoría técnicoadministrativa del artículo, revisión crítica del manuscrito, y aprobación final del manuscrito.

Financiamiento: Autofinanciado.

Conflicto de interés: Los autores declaran no tener conflictos de interés.

Recibido: 22 de octubre 2019

Aprobado: 26 de noviembre 2019

Correspondencia: Alexander H. Román Meza.

Dirección: Cl. Tarata 493, La Perla - Callao.

Teléfono: 949071089 - 4200066

Correo:alexrom2490@gmail.com 


\section{REFERENCIAS BIBLIOGRÁFICAS}

1. Jiménez L, Montero J. Medicina de urgencias y emergencias: Guía diagnóstica y protocolos de

actuación. 5a ed. Barcelona: Elsevier; 2015.

2. Borruel M, Martínez A, Estabén V., Morte A. Manual de Urgencias Neurológicas. Teruel: Servicio de Urgencias del Hospital Obispo Polanco; 2013.

3. Mercado T. Correlación clínicotomográfica en la evolución del Trauma Craneoencefálico en el Hospital Antonio Lenin Fonseca, octubre 2016. Tesis de Especialidad. Managua, Nicaragua. Facultad de Ciencias Médicas, Universidad Nacional Autónoma de Nicaragua; 2017. 73 pp.

4. Zarranz J. Neurología. 6a ed. Barcelona: Elsevier; 2018.

5. del Cura J, Pedraza S, Gayete A. Radiología esencial. 2a ed. Madrid: Editorial Médica Panamericana; 2018.

6. Lobo E. Manual de Urgencias Quirúrgicas. 5a ed. Madrid: Imprenta Pedragosa; 2016.

7. Román A. Características epidemiológicas y patologías halladas po tomografía computada cerebral en adultos atendidos en emergencia. Tesis Lima, Perú. Facultad de Medicina, Universidad Nacional Mayor de San Marcos; 2012. $91 \mathrm{pp}$.

8. Pope T, Harris J. Radiología en Emergencias Médicas Harris \& Harris. 5a ed. Medellín: Editorial Amolca; 2014.

9. Onofre J, Mancilla A. Utilidad actual de la radiografía simple en el diagnóstico de fracturas de cráneo. An Rad Mex. 2010; 2(1): 73-75.

10. Ortega E. Técnica radiológica para pacientes con Traumatismo Craneoencefálico con Uso de Rayos X. Tesis. Loja, Ecuador. Área de la Salud Humana, Universidad Nacional de Loja; 2013. 45 pp.

11. Torre J. Diagnóstico por imágenes en pacientes con Traumatismo encefalocraneano en el Tópico de Emergencia de Cirugía del Hospital Carlos Lanfranco La Hoz de Julio 2016 - Noviembre 2016. Tesis. Lima, Perú. Facultad de Ciencias de la Salud, Universidad Privada San Juan Bautista; 2017. 52 pp

12. Avilés C, Ayala J, Bermeo J. Hallazgos tomográficos en pacientes con traumatismo craneoencefálico. Departamento de Imagenología. Hospital Vicente Corral Moscoso. Cuenca, julio - diciembre 2012. Tesis. Cuenca, Ecuador. Facultad de Ciencias Médicas, Universidad de Cuenca; 2013. 74 pp.

13. Osborn A. El Encéfalo: Diagnóstico por imagen, patología y anatomía. Madrid: Editorial Médica Panamericana; 2018.

14. Varela A, Martínez C, Muñoz R, Torres R, Orellana F, Lamus L, Herrera P. Algoritmo para la tomografía secuencial de cráneo en pacientes con traumatismo encéfalocraneano. Rev Chil Neurocirugía. 2016; 42(1): 24-30.

15. Belduma V. Traumatismo craneoencefálico: Diferencias tomográficas entre el hematoma epidural y subdural para el diagnóstico precoz de sus complicaciones. Tesis. Machala, Ecuador. Unidad Académica de Ciencias Químicas y de la Salud, Universidad de Machala; 2019. 23 pp.

16. Morales H. Utilidad de la Tomografía axial computarizada para la detección de hematomas epidurales y subdurales en pacientes de 40 a 60 años de edad en el Hospital "Enrique Garcés" en el periodo de agosto 2015 a diciembre 2015. Tesis. Quito, Ecuador. Facultad de Ciencias Médicas, Universidad Central de Ecuador; 2016. 79 pp.

17. Rodríguez M, Dosouto V, Rosales Y, Musle M, González Y. Valor de la tomografía axial computarizada para el diagnóstico precoz del traumatismo craneoencefálico. MEDISAN. 2010; 14(6): 767-773.

16. Guamán V. Utilidad de la Tomografía Simple de Cráneo para la Detección del Hematoma Subdural Crónico en pacientes de 40 A 60 años que acuden al servicio de Imagen del Hospital de Especialidades Eugenio Espejo de la ciudad de Quito en el periodo Julio a Diciembre del 2015. Tesis. Quito, Ecuador.
Facultad de Ciencias Médicas, Universidad Central del Ecuador; 2016. 113 pp. 19. Remón Cr Pernía L, Corrales $N$ castañeda C Tomografía axia computarizada en traumatismos encefalocraneanos. Experiencia en 6 años: Enero 2006-Diciembre 2012. Multimed. 2013; 17(2): 64-80.

20. Osborn A. Cerebro. Serie Diagnóstico por Imagen. 2a ed. Madrid: Marbán; 2011.

21. Sierra E, León M, Rodríguez E, Pérez L. Caracterización clínico-quirúrgico, neuroimagenológico y por neuromonitorización del trauma craneoencefálico en la Provincia Matanzas 2016-2018. Rev Med Electron. 2019; 41(2): 368-381.

22. Tandazo D. Determinación imagenológica de la lesión cerebral mediante tomografía axial computarizada en pacientes con trauma craneoencefálico ingresados en el Hospital Isidro Ayora. Tesis. Loja, Ecuador. Área de la Salud Humana, Universidad Nacional de Loja; 2014. 95 pp.

23. Cuya C. Traumatismo encefalocraneano mediante tomografía axial computarizada en pacientes atendidos en el Centro Médico Naval Cirujano Mayor Santiago Távara periodo julio 2013-2015. Tesis. Lima, Perú. Facultad de Medicina Humana y de Ciencias de la Salud, Universidad Alas Peruanas; 2016. $42 \mathrm{pp}$.

24. Fernández F, Timbe C. Incidencia de lesiones causadas por traumatismo cráneo encefálico diagnosticadas por tomografía en pacientes del Hospital Homero Castanier Crespo de Azogues, período enero - diciembre del 2017. Tesis. Cuenca, Ecuador. Facultad de Ciencias Médicas, Universidad de Cuenca 2019. 84 pp.

25. González R, Garbey B, Valdés O. El ABC del accidente cerebro vascular en la tomografía computarizada de cráneo. Rev Cub Med Intensiva y Emergencias. 2018; 17(1):19-35

26. Fernández A, Aladrén J, Tejada H, Cruz G, Angel L, Seral P, Artal J, Marta J. Signos predictores de crecimiento precoz de la hemorragia intracerebral en la tomografía computarizada sin contraste y mortalidad. Rev Neurología. 2018; 67(7): 242-248.

27. Rodríguez S, Domínguez E, Bolaños S. Tomografía axial computarizada en las enfermedades cerebrovasculares hemorrágicas. Panorama Cuba y Salud. $2011 ; 6(1): 139-141$

28. Reyes Y. Tomografía computarizada y el diagnostico de patología cerebrovascular hemorrágica. Tesis. Guayaquil, Ecuador. Facultad de Ciencias Médicas, Universidad de Guayaquil; 2012. 106 pp.

29. de Alba J, Guerrero G. Evento vascular cerebral isquémico: hallazgos tomográficos en el Hospital General de México. An Rad Mex. 2011; 3(1): 161166

30. Blanco M, Arias S, Castillo J. Diagnóstico del Accidente Cerebrovascular Isquémico. Medicine. 2011; 10(72): 4919-4923.

31. Herrera A. Signos radiológicos presentes en tomografía computada simple en pacientes con accidente cerebrovascular isquémico. Instituto Nacional de Ciencias Neurológicas, Octubre a Diciembre del 2017. Tesis. Lima, Perú. Facultad de Medicina, Universidad Nacional Mayor de San Marcos; 2018. 103

32. Pitot L. Comparación de imágenes entre secuencia convencional y secuencia de difusión en la resonancia magnética nuclear del accidente cerebrovascular isquémico agudo. Centro Diagnóstico Osteoperú, Lima-2017. Tesis. Lima, Perú. Facultad de Medicina, Universidad Nacional Mayor de San Marcos; 2018. 84 pp.

33. Reyes I, Rivas C, Robles C. Tomografía Tomografía Axial Computarizada vs. Resonancia Magnética como método de estudio de la Enfermedad Cerebro Vascular Isquémica Aguda. Naguanagua, Estado Carabobo, Año 2013. Tesis. Carabobo, Venezuela. Facultad de Ciencias de la Salud, Universidad de Carabobo; 2013. 25 pp. 